\title{
ARRIBA DEL CERRO O SOBRE EL HUMEDAL: PRODUCCIÓN DE NATURALEZA Y EXPANSIÓN INMOBILIARIA EN CIUDADES MARINAS Y FLUVIALES. EL CASO DE VALPARAÍSO Y VALDIVIA, CHILE*
}

\author{
UP THE HILL OR OVER THE WETLAND: PRODUCTION OF NATURE \\ AND EXPANDING REAL ESTATE IN MARINE AND FLUVIAL CITIES. \\ THE CASE OF VALPARAÍSO AND VALDIVIA, CHILE
}

\author{
Rodrigo Hidalgo $^{* *}$, Laura Rodríguez ${ }^{* * *}$ y Voltaire Alvarado ${ }^{* * * * *}$
}

\begin{abstract}
El emplazamiento urbano de características fluviales o marinas ostenta ciertas condiciones de sitio que hacen de estos un complejo campo de investigación para la Geografía no solo por la relación entre paisaje y habitar, sino por la ocupación de zonas desechables para el mercado como nichos grandes para negocios inmobiliarios.

Desde las ciudades de Valparaíso y Valdivia, este trabajo describe la relación entre las quebradas que rodean a la primera y los humedales que circundan a la segunda, y los conjuntos de habitaciones sociales presentes en ellas. Considerando su rol en políticas públicas, se revisa su situación respecto de estos elementos propios del entorno desde la producción de naturaleza y su apertura a la modificación del medio en favor de la renta.

La metodología se basa en la consideración del Índice de Desarrollo Socioeconómico (IDS) presente en ambas ciudades, los conjuntos de vivienda social y la relación espacial de proximidad existente entre estos y los elementos del medio ya individualizados. Desde ellos, se presentan mapas y hojas de ruta para reconocer a la producción de naturaleza como eje en la capacidad de reproducción que la renta tiene sobre el espacio, sin importar su condición primigenia. Los resultados obtenidos indican una ocupación situada en la condición de clase y sitio, además de la incidencia del paisaje en la construcción social del valor.
\end{abstract}

Palabras claves: Producción de naturaleza, condición de sitio, extractivismo inmobiliario, ciudades marinas y fluviales.

River or marine coastal urban settlement has certain site conditions, which make them a complex field of research for Geography. This is not exclusively just due to the relationship between landscape and dwelling, but also because the occupying neglects areas, by the market, as large niches for real estate businesses.

This research describes the relationship between social housing and its closeness to brooks and wetlands in the city of Valparaiso $y$ Valdivia. Considering its relevance in public policies, it reviews these environment components, as a production of nature approach and its willingness to the open it up in favor of income.

The Index of Socioeconomic Development, present in both cities, is the selected data. Besides, social housing conglomerates and proximity to the environmental components (as brooks and wetlands) are considered. Finally, maps are display to recognize the production of nature as the main component over rent increasing on the space, regardless of its initial condition. The results obtained indicate a located occupation about condition of class and site, as well the incidence of landscape in the social construction of value.

Key words: Nature production, site condition, real estate extractivism, marine and river cities.

\section{Introducción}

Tanto Valparaíso como Valdivia han sido edificadas a partir de la producción de naturaleza. El concepto asociado a esta afirmación, a pesar de que su uso tiene una masificada presencia en las ciencias sociales, no ha sido definido o reconocido en tanto organiza las discusiones críticas contenidas en distintos volúmenes científicos, académicos o profesionales. Para los fines que más adelante se detallan, la producción será entendida como el resultado material de varios procesos, transacciones, conflictos y rupturas acontecidas entre las dimensiones telúricas y sociales, donde

\footnotetext{
* Resultado Proyecto FONDECYT No 1150360.

** Pontificia Universidad Católica de Chile, Instituto de Geografía. Macul, Santiago de Chile. Correo electrónico: rhidalgd@uc.cl

*** Universidad Austral de Chile, Instituto de Arquitectura y Urbanismo. Valdivia, Chile. Correo electrónico: 1rodriguez@uach.cl

***** Pontificia Universidad Católica de Chile, Instituto de Geografía. Universidad Academia de Humanismo Cristiano, Escuela de Geografía. Macul, Santiago de Chile. Correo electrónico: vcalvarado@uc.cl
} 
la naturaleza es el objeto de transformación que expresa esta secuencia.

Las razones que justifican esta temprana definición organizan un debate esencial, pues sin ella sería improcedente describir lo natural como un producto de la acción social, y a lo inmobiliario como artefacto espacial enfrentado a la necesidad por cubrir demandas sociales variopintas, donde una es la vivienda.

Este trabajo propone establecer un marco para discutir acerca de la producción de naturaleza y la condición espacial frente a la expansión inmobiliaria de la vivienda social en los casos de Valparaíso y Valdivia, Chile. Ambas zonas urbanas albergan una cantidad significativa de conjuntos habitacionales con interés social edificados más allá de los límites de lo normativo y, por cierto, de aquellas barreras que la naturaleza ha representado a lo largo de su historia. Así como en Valparaíso la presencia de los cerros que envuelven a la bahía es símbolo y postal de su emplazamiento, también lo es de su precariedad como espacio de habitar para las clases populares. De la misma forma, pero rodeando y rellenando humedales, Valdivia exhibe una larga tradición en la generación de nuevos espacios para el asentamiento, asumiendo no solo aquellos que son generados por sus propia instrumentalidad políticoterritorial, sino que por la necesidad de habitación. En ambos, las soluciones habitacionales urbanizan en zonas inadecuadas para la fijación de una habitación permanente, al servicio de las dinámicas expoliativas del mercado inmobiliario chileno.

Para estos fines se ejecutan dos procesos para sustentar las cuestiones planteadas. Hacia el final del trabajo se propone una reconstitución de los conceptos ofrecidos al inicio, refrendando las posibilidades hacia un examen complejo de la relación entre el ser humano, en tanto agente de transformación espacial clave, y lo natural. Si es que es posible definir la naturaleza en el marco del mercado inmobiliario.

\section{Metodología}

El trabajo realizado obtuvo sus fuentes empíricas desde dos universos diferentes. El primero correspondió a la realización de un catastro de las viviendas sociales construidas desde 1980 hasta 2016 generado a partir de información obtenida en los registros centrales y regionales del Ministerio de
Vivienda y Urbanismo. La localización se efectuó a partir de la revisión de planos y mapas en las Direcciones de Obras Municipales, calibrado con trabajo en terreno.

Para construir los datos del Índice de Desarrollo Socioeconómico -IDS- se utilizó la información del Censo 2002 y el precenso de 2016, con el fin de ajustar la muestra a un nivel de precisión interpretativa concreta, considerando las limitantes que el Censo de 2012 y su declaratoria de invalidez como instrumento y base de datos ${ }^{1}$. Este ajuste consistió en el uso de las manzanas censales establecidas para la consulta de 2002 y la información de carácter social y económica del precenso de 2016, ajustándose así ambas dimensiones al modelamiento de las ciudades de Valparaíso y Valdivia.

El procedimiento del cálculo del IDS fue tomado a partir del Índice de Desarrollo Humano -IDH-, metodología propuesta por el Programa de Naciones Unidas para el Desarrollo -PNUD- (PNUD/ MIDEPLAN, 2000). El IDS se construyó basado en tres tipos de indicadores o dimensiones: bienes suntuarios (auto particular, lavadora, camioneta particular, microonda, moto particular, refrigerador, teléfono red fija, televisión a color, videograbador, internet y televisión por cable), nivel de estudio de la población de más de 25 años (humanidades, educación media, centro de formación técnica, instituto profesional y universidad), y calidad de la vivienda (techo, piso y pared, en función de los materiales utilizados en su construcción). Cada una de las variables detalladas fueron estandarizadas bajo la siguiente relación

$$
\frac{\text { valor máximo - valor mínimo }}{\text { valor de la variable - valor mínimo }}
$$

Por cada manzana, finalmente, se sumaron los tres valores estandarizados de cada dimensión considerada, obteniéndose así el IDS para cada zona censal de las comunas en estudio. Los valores más altos expresan mayores niveles de desarrollo socioeconómico. El conjunto de datos para realizar dicho cálculo se efectuó mediante Redatam-G4, definido como un sistema informático para recuperar datos censales en áreas menores (CEPAL/ CELADE, 2003).

Desde esta síntesis se calcularon dos líneas de IDS. La primera de ellas referida al IDS Global, que contiene las categorías de nivel bienes suntuarios en 
el hogar, nivel educacional y calidad de la vivienda a nivel de manzanas censales desplegadas por cada comuna en cuestión. La segunda línea se compone de mostrar el detalle del IDS de vivienda -debido al objeto de estudio- aplicado a los conjuntos habitacionales que forman parte del análisis ofrecido por el trabajo, como se expresa en la Tabla 1.

Finalmente, a partir de la medición de distancias euclidianas entre los elementos del medio individualizados en quebradas para Valparaíso y humedales para Valdivia, se establece una relación lineal entre ambos indicadores, con el propósito de reconocer el resultado espacial de la producción de naturaleza.

\section{La producción de naturaleza como articulación del espacio urbano}

Neil Smith en su obra La Producción de Naturaleza enfatiza respecto de la diatriba presente en la caracterización de lo natural como algo producido, asunto que el autor resuelve a renglón seguido, señalando que "este sustrato material es cada vez más el resultado de la producción social" (Smith, 2006:13).

Aquí la técnica al servicio de la acumulación de capital se convierte en un elemento clave en el espacio de la producción de naturaleza, que es intrínsecamente espacio geográfico. El mismo Smith lo describe con base en las escalas que la primera y segunda naturaleza proponen, cuyo sentido espacial no es otro que el valor de cambio materializado en objetos modificadores del entorno inicial:

Sin introducir el valor de cambio en la naturaleza, la relación entre la primera y la segunda naturaleza no puede ser entendida en su forma concreta. Podría resultar difícil moverse más allá de la ambigua, limitada, y eventualmente ideológica pretensión de que, por un lado, la naturaleza es social, mientras que, por el otro, la sociedad es natural (Smith, 2006:32).

Bajo esta lógica, y hace más de un siglo, Elisée Reclus se había referido a la alteración de la naturaleza, tipificándola en dos grandes grupos: una primera, más bien prístina, que estaría ajena a la intervención humano-social; y una segunda, compuesta por toda unidad espacial donde el cuerpo social interviniese para asentarse, sobrevivir o expandirse (Reclus, 1875). Ambas ideas, distantes pero adyacentes a la vez, sugieren como elemento común a la técnica como palanca en la intervención de la tierra. Por ello, la elaboración de Smith y el énfasis en el valor de cambio rehabilitan el categórico juicio que el sabio ruso Piotr Kropotkin elaborara acerca del poder de la ciencia al servicio de la modernización, discutiendo su efectivo rol en la revolución y transformación social que no tenía entre sus proyectos un fin colectivo, más que el de la acumulación de riqueza por sí misma (2016).

Es posible establecer, entonces, que la producción de naturaleza dialoga de forma concreta con la teoría de la renta y la acumulación vertidas en la obra de Friedrich Engels estableciéndose también un nexo con la idea de la vivienda como dispositivo de modernización y microrriqueza, asuntos que el citado autor aborda en El Problema de la Vivienda (2015 [1873]). Es, entonces, la propiedad y su tenencia la que detona esta problemática de usos de lo natural, particularmente en la forma que Engels observa las tensiones decimonónicas de Inglaterra y la configuración de clase con que se expanden las ciudades en la primera gran crisis de la que es testigo el autor y que Henri Lefebvre destaca

Tabla 1. Materiales, métodos y productos asociados a las áreas de estudio

\begin{tabular}{lll}
\hline \multicolumn{1}{c}{ Materiales } & \multicolumn{1}{c}{ Procedimientos } & \multicolumn{1}{c}{ Productos } \\
\hline $\begin{array}{l}\text { Censo 2002/Precenso 2016 } \\
\text { Conjuntos de vivienda } \\
\text { social-Valparaíso }\end{array}$ & Cálculo IDS Global a nivel comunal & Indicadores organizados según distancia \\
57 conjuntos & Cálculo IDS Vivienda con relación a & $\begin{array}{l}\text { Tabla de distancias entre quebradas y con- } \\
\text { juntos de viviendas sociales 0 a 1.300 metros } \\
\text { (euclidiana) }\end{array}$ \\
$\begin{array}{l}\text { Conjuntos de vivienda } \\
\text { social-Valdivia }\end{array}$ & información IDS Global & $\begin{array}{l}\text { Tabla de distancias entre quebradas y con- } \\
\text { j8 conjuntos }\end{array}$ \\
\hline
\end{tabular}

Fuente: elaboración propia. 
Ciento sesenta y siete años más tarde, este "ambiente", este "clima", ¿realmente han cambiado? Basta con haber estado algún tiempo en una ciudad norteamericana para responder, aun si en las ciudades europeas la estrategia de clases dominantes ha transportado a los trabajadores a los suburbios en donde se estacan, aislados, en la paz represiva (Lefebvre, 2014:20).

De la misma forma, pero sin tocar el concepto de naturaleza como un producto, Karl Marx ensaya algunas de estas premisas en la conocida fórmula trinitaria, que corresponde a la relación entre la tierra, como sustrato de base para la realización de toda actividad humana; el capital, contenido no solo en la inversión monetaria sobre las ocupaciones sino también en la técnica e innovación para la producción; y, finalmente, el trabajo, correspondiente a la mano de obra (Marx y Engels, 1998 [1894]). Entre ellos, que conforman los factores de la producción, se genera la acumulación de capital y maximización de la renta, precisamente desde la precarización en el pago por la mano de obra.

Esta renta, obtenida precisamente desde el plusvalor, coloca a la propiedad de las cosas como un bien supremo en importancia respecto de otros. La producción de naturaleza, entonces, forma parte del proyecto modernizador de la burguesía, donde la técnica es la productora de la mercancía como resultado fáctico del monopólico ejercicio del saber y el hacer. Para la generación de renta desde la tierra, es la naturaleza la que debe ser alterada hasta convertirse en un seductor polígono, capaz de atraer no solo a la inversión público-privada, sino que también de convencer a las personas de habitarlo.

Con el trabajo inconcluso de Friedrich Engels sobre la ciencia, ya aparecía el debate pertinente a una naturaleza producida a partir de la técnica, dejando en claro que la ciudad sería la consumación en la sofisticación de la misma, superando así los paradigmas de inamovilidad de la naturaleza que, según el autor, habían secuestrado para sí el conocimiento crítico respecto de la dialéctica entre lo social -la producción y acumulación de capital-y lo natural -aquello que está dado en la superficie de la Tierra-(Engels, 2017 [1971]). Lo anterior se refuerza en el sentido de la condición de sitio que, desde la geografía, considera a la producción de naturaleza y entorno a partir de dos dimensiones: una espacial, capaz de "tipificar las condiciones de asentamiento actual con sus características otorgadas por los datos del medio natural y las creaciones humanas para adaptarlo a sus necesidades locales y relacionales"; y otra temporal, sostenida en "anticipar el comportamiento de las condiciones de asentamiento para aceptar programas de desarrollo y nuevas actividades en el futuro" (Gray de Cerdán, 1987:68).

Entonces, la invención de la naturaleza como motivación para la ocupación del espacio representa la culminación de un proceso de transformación del entorno. Más allá de la dialéctica entre determinismo y posibilismo geográficos, la creación de cierta artificialidad espacial para la gestión y reproducción de la renta del suelo es, sin duda, un escenario inédito aún para las tendencias geográficas más críticas, algo que es considerado por Aliste y Musset respecto del uso de tierras agrícolas como un polígono vacío a la hora de urbanizar (2014:98). Un claro ejemplo de ello está en la construcción de conjuntos residenciales adosados litoralmente a cuerpos de agua urbano-interiores, los que a partir de un tratamiento constante entregan para sus propietarios hermosas y cristalinas aguas durante todo el año. En Chile este negocio ha crecido y no ha estado exento de acusaciones por secar acuíferos, aportar a la desertificación y el aumento de insectos en épocas invernales, siendo el móvil la invención de lo natural al servicio de mayor rentabilidad y plusvalor, constituyendo un tercer escalón en la racionalidad de la producción de naturaleza, ahora inventada con fines rentistas (Hidalgo et al. 2016).

Las discusiones anteriores están presentes en el debate latinoamericano acerca de la producción y la renta extractiva del suelo urbano. Desde el mismo piso, la renta urbana en la región ha sido trabajada por Samuel Jaramillo y Alberto Lovera. El primero establece una relación crítica entre las mutaciones del Estado latinoamericano como proyecto nacional, y la necesidad de validarse espacialmente en la producción habitacional de viviendas populares, estableciendo un proceso estatal de promoción de la propiedad de la tierra a escala humana y, desde aquí, anunciar que el nicho rentista sería proveído desde los fiscos nacionales (Jaramillo, 2001). Lovera define, por su parte, un campo de acción más amplio, señalando el rol del "medio ambiente construido" como base en las acciones de modernización y transformación inmobiliaria del paisaje urbano, considerando el papel que juegan los agentes espaciales como central en el modelamiento habitacional, por ejemplo (Lovera, 2013:3). 


\section{Los cerros que envuelven a la bahía: vivienda encaramada en Valparaíso}

Valparaíso representa un ejemplo vívido de la adjetivación espacial contemporánea. Primero, por su inscripción como patrimonio de la humanidad por UNESCO en 2003 convirtiéndola en postal obligada para el turismo internacional; y segundo, porque alberga elementos morfológicos excepcionales que rodean la bahía, como es el conjunto de cerros formados por una proyección transversal de la cordillera de la Costa. Un entorno único, que sin embargo presenta una alta exposición a eventos sísmicos, inundaciones por tsunami, remoción de laderas en la zona de los cerros e incendios forestales con alta periodicidad para los últimos cinco años (Indirli y Apablaza, 2010). Estos cerros registran altitudes de hasta 302 metros sobre el nivel del mar, como es el caso del sector Rodelillo que acoge una alta densidad residencial vertical de viviendas sociales, cuya exposición a incendios prolongados durante los meses de verano consume toda informalidad habitacional existente en la zona para proveer, posteriormente, de edificios con servicios mínimos urbanos normalizados tan básicos, pero anteriormente ausentes, como agua potable y arranque eléctrico.

Por otro lado, la condición patrimonial de las zonas centrales del municipio de Valparaíso, sumado al alto costo y situación de clase que representa Viña del Mar desde el centro y hacia los cerros, impide edificar complejos habitacionales de alta densificación y con vocación social al interior de las ciudades (Marín et al. 2017). Es en el marco de estos cerros que se configura el paisaje de viviendas encaramadas en secas laderas y caminos sin pavimento que, al mismo tiempo, concentran a las clases trabajadoras la metrópolis porteña. Aquí, la naturaleza no solo ha sido adaptada para la producción de sendos conjuntos habitacionales irregulares, sino que también ha sido inventada como alternativa para acoger operaciones inmobiliarias accesibles para los sectores populares, por medio de las políticas residenciales de subsidios impulsadas por el Estado chileno desde la década de 1980 a la actualidad (Zunino y Hidalgo, 2009).

Claro, se manifiesta hacia el observador externo como un lindo paisaje, apreciándose solo una parte del puerto, mas no la sección que habitan las familias pobres de la ciudad litoral. Las formas de producción espacial y la edificación de conjuntos habitacionales de carácter social configuran un otro paisaje, el de las precariedades obliteradas pero tangibles, respecto de las que se cimientan aún en el siglo XXI soluciones residenciales autogestionadas ante la expoliación inmobiliaria de las zonas urbanas. Pero es esta urbanización espontánea la que abre el camino hacia los nichos de renta, pues esta vivienda informal impulsa la ejecución de políticas para la presencia y acceso a servicios de salud, educación y comercio en general en zonas donde, inicialmente, no estarían las condiciones para recibirlas. Con este impulso, la producción de naturaleza es concomitante a la urbanización masiva para las soluciones habitacionales, pero luego se entrega hacia la elitización de estos sectores en función de los atractivos ya señalados (Borsdorf e Hidalgo, 2013).

\section{Arriba del cerro está la vivienda social}

El marco de datos utilizado corresponde a las bases censales para los años 2002 y 2012, considerando el cálculo de población por manzana e Índice de Desarrollo Socioecómico -IDS-. En función de ellas, adicionando la localización de las viviendas sociales en Valparaíso, se ingresan las quebradas como sectores expuestos y sensibles para la producción de naturaleza. Si se consideran todas las quebradas de la zona, se obtienen 1.032 entidades espaciales de este tipo, dibujando un panorama que por sí solo acusa la condición espacial que Valparaíso ofrece hacia la urbanización, como aparece en la figura a continuación.

Por otro lado, el cálculo del IDS se realizó sobre las manzanas censales de 2002 y 2016 y consolidó una muestra homogénea en función de la perspectiva de precariedad en las habitaciones asentadas en las quebradas. Es así como la relación de distancia entre el elemento natural y los conjuntos de vivienda social articulan la producción de naturaleza, la que se coloca al servicio de la expansión habitacional.

En la Tabla 2 se encuentran los resultados del IDS de acuerdo con la distancia entre manzanas y quebradas. Como primera aproximación al producto cartográfico, la tabla muestra que existiría una relación lineal entre ellas. Esto se podría deber a que las manzanas ubicadas en el centro norte son aquellas con mayor puntuación acumulada -Tabla 2respecto del índice al localizarse hacia la comuna de Viña del Mar que, por conurbada que se encuentre al Área Metropolitana de Valparaíso, concentra una mejor calidad de vida que sus vecinas al norte y sur. 


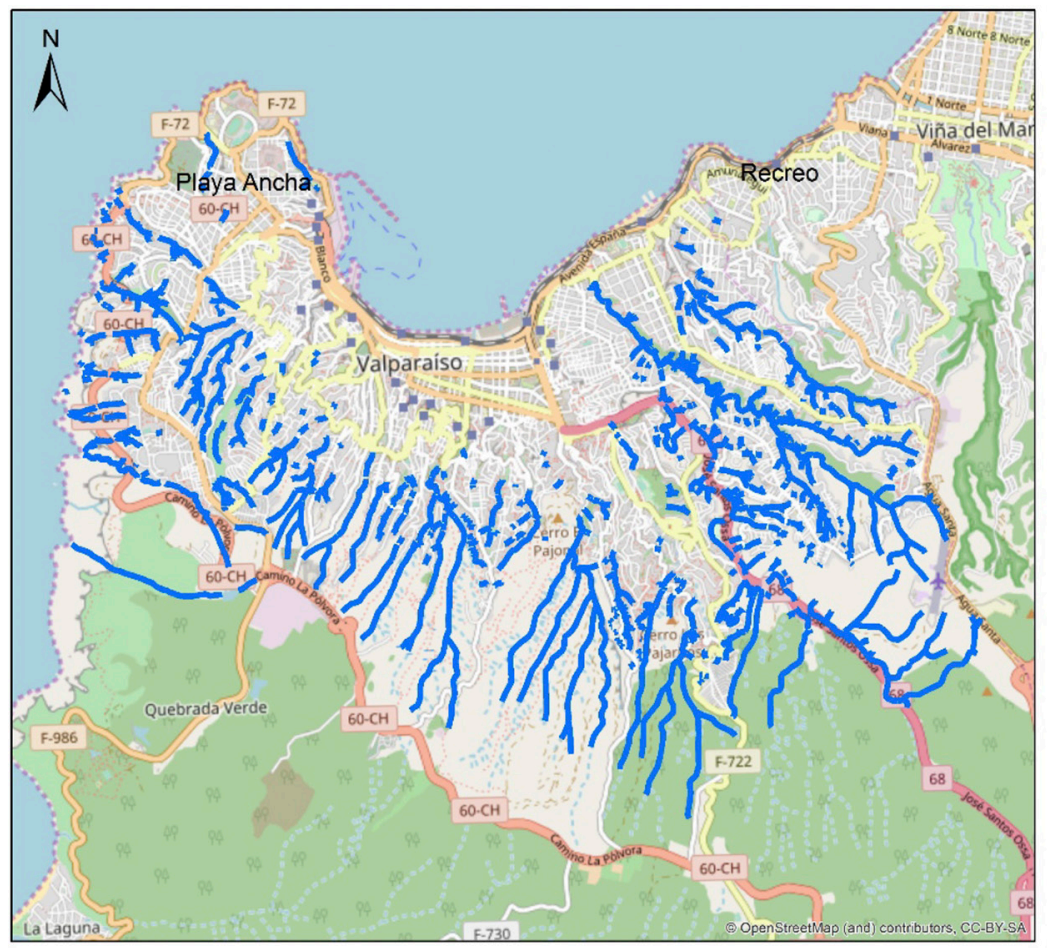

\section{Leyenda}

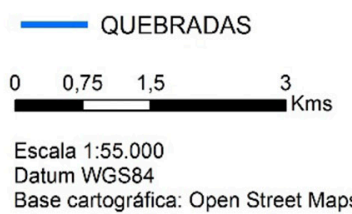

Figura 1. Distribución de las quebradas en la comuna de Valparaíso.

Fuente: elaboración propia con base en datos IDE-Chile 2017.

Tabla 2. Promedio IDS Global por manzana censal e IDS por vivienda respecto de las quebradas, Área Metropolitana de Valparaíso

\begin{tabular}{ccc}
\hline $\begin{array}{c}\text { Distancia desde las manzanas } \\
\text { a la quebrada más cercana [m] }\end{array}$ & $\begin{array}{c}\text { Promedio de IDS } \\
\text { GLOBAL }\end{array}$ & $\begin{array}{c}\text { Promedio de } \\
\text { IDS VIVIENDA }\end{array}$ \\
\hline $0-100$ & 1,919 & 0,921 \\
$100-200$ & 2,010 & 0,898 \\
$200-300$ & 2,047 & 0,877 \\
$300-400$ & 2,026 & 0,858 \\
$400-500$ & 2,004 & 0,833 \\
$500-600$ & 2,009 & 0,856 \\
$600-700$ & 2,001 & 0,865 \\
$700-800$ & 1,999 & 0,875 \\
$800-900$ & 2,033 & 0,847 \\
$900-1000$ & 2,085 & 0,887 \\
$1000-1100$ & 2,201 & 0,933 \\
$1100-1200$ & 2,366 & 0,995 \\
$1200-1300$ & 1,528 & 1,000 \\
\hline
\end{tabular}

Fuente: elaboración propia con base en datos Censo 2002/bases censales 2016.

Caso contrario el de Valparaíso, donde las quebradas tienen una disposición sur más bien asociada a las viviendas sociales de la zona. Este promedio de distancias expresado en metros explica la inevitable asociación entre las viviendas asentadas en las quebradas y el bajo índice de desarrollo socioeconómico. La precariedad asociada a la producción de naturaleza y las vanguardias urbanizadoras cumplen con el sucio trabajo de abrirle cancha al nicho grande inmobiliario. 

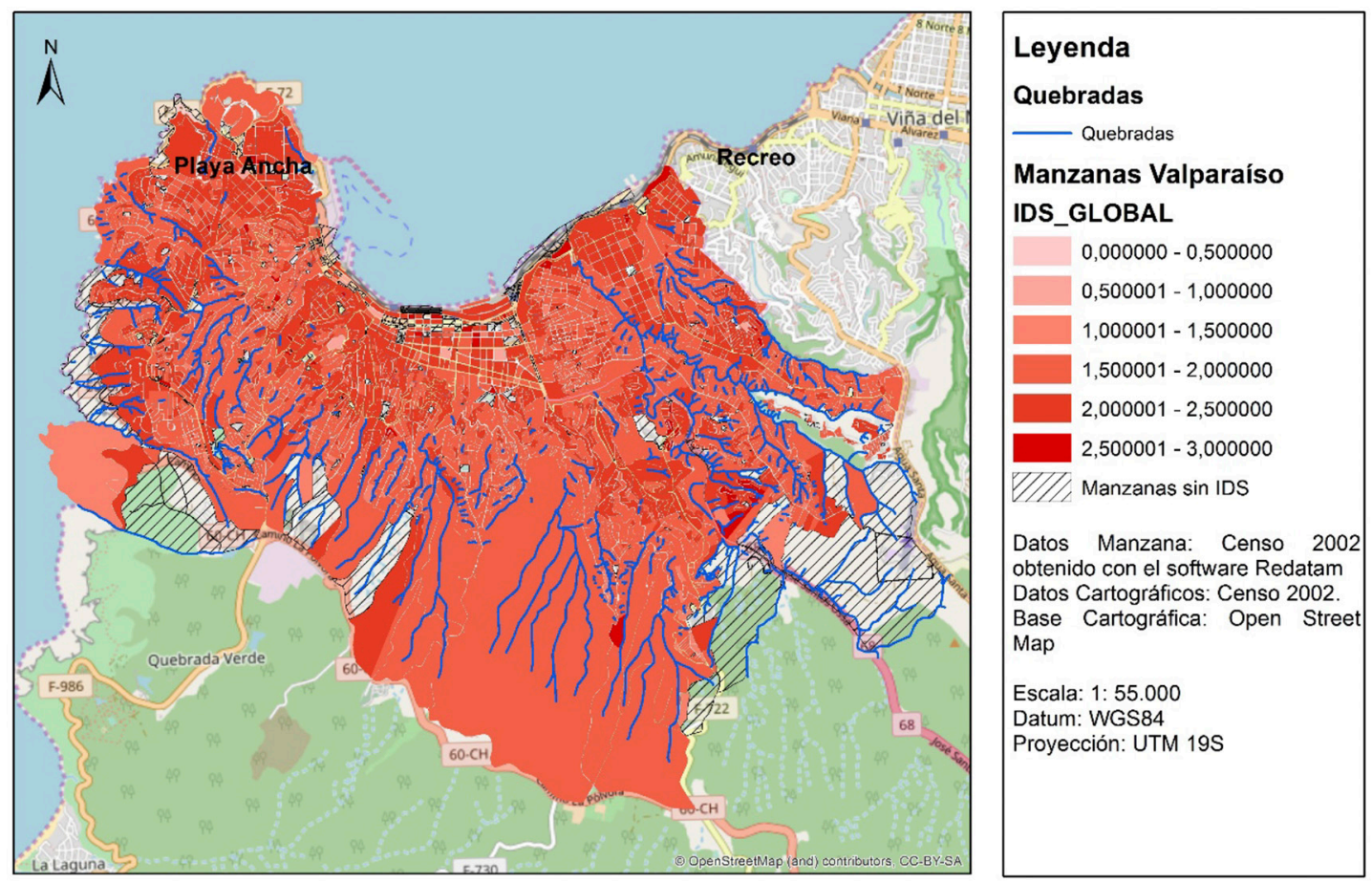

Figura 2. Distribución IDS Global y quebradas, Área Metropolitana de Valparaíso.

Fuente: elaboración propia con base en datos IDE-Chile 2017.

En el mapa y tabla anteriores se manifiesta una relación entre la distancia a las quebradas y el índice de desarrollo socioeconómico. A estos indicadores se adicionan los conjuntos de vivienda social presentes, que en la zona suman 57 complejos habitacionales.

Estas viviendas exhiben una tendencia conforme de concentración de acuerdo con el IDS, poblando además sectores masivos de quebradas y conjuntos sociales, como los ubicados en el camino La Pólvora, hacia el centro norte de la comuna, y el sector Playa Ancha hacia el sur de la bahía, tal como lo muestra la Figura 3.

A la elocuencia de estos datos, se agrega la alta concentración de conjuntos sociales emplazados en los tramos más cercanos a los cerros. Si en los rangos de ambos IDS figuran ciertos matices, que podrían explicarse por el uso turístico de los cerros que envuelven a la bahía de Valparaíso, se produce un cambio concreto al agregar la densidad de viviendas que contienen estas agrupaciones habitacionales. La siguiente tabla no solo acusa esta condición, sino que valida la tesis que la naturaleza es alterada de forma constante para la creación de espacios urbanos, donde el nuevo propietario constituye la reproducción expoliativa de las soluciones habitacionales (Hidalgo et al. 2017).

\section{Ocupación, vivienda y relleno: producción de naturaleza en Valdivia}

La convención histórica indica que la ciudad de Valdivia ha sido edificada sobre los humedales desde tiempos pretéritos (Adán, et al. 2017). No obstante, la relación con la naturaleza ha sido, en esencia, cambiante y tumultuosa. Si por un lado el paisaje natural representa el atributo más notable de la ciudad, por el otro constituye una fuente de tribulaciones para las clases expoliadas, siendo la naturaleza un factor de diferenciación relevante en la producción inmobiliaria.

Como se planteó antes, las investigaciones histórico-arqueológicas evidencian la presencia constante de cuerpos de agua, los que se proyectan más allá del importante río Calle-Calle y el sistema fluvial que articula su curso. Los humedales, despreciados inicialmente como pantanos y luego valorados desde lo legal y lo científico, han sufrido extendidas modificaciones con la transformación de Valdivia: estos han sido modificados, empequeñecidos, rellenados 


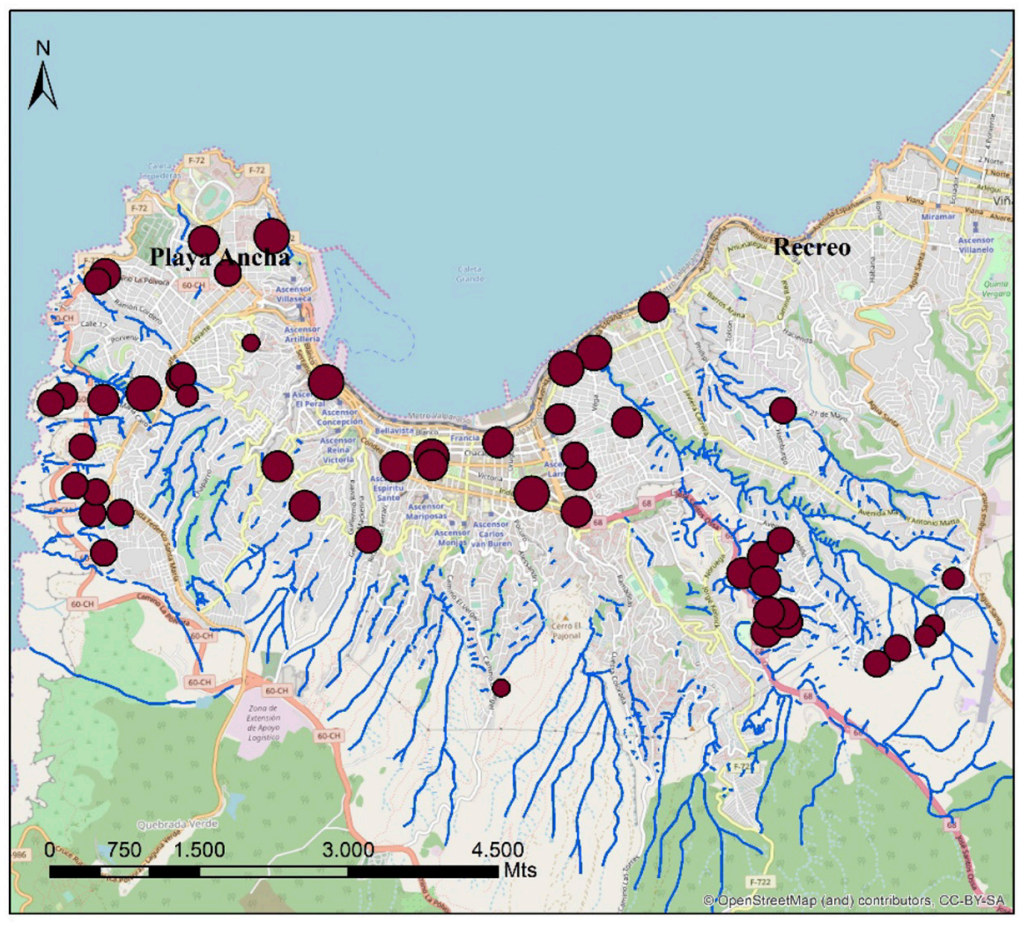

\section{Leyenda}

Viviendas Sociales IDS_GLOBAL

- $1,551646-1,800000$

- $1,800001-2,000000$

2,000001-2,200000

2,200001-2,400000

2,400001-2,600000

\section{Quebradas}

\section{Quebradas}

Datos IDS: Censo 2002 obtenido con el software Redatam

Datos Viviendas Sociales: Proyecto Fondecyt

Base Cartográfica: Open Street Map

Escala: 1: 50.000

Datum: WGS84

Proyección: UTM 19S

Figura 3. Distribución IDS, quebradas y conjuntos de viviendas sociales, Área Metropolitana de Valparaíso.

Fuente: elaboración propia con base en datos IDE-Chile 2017.

Tabla 3. Relación de distancia y densidad de conjuntos habitacionales respecto de las quebradas, Área Metropolitana de Valparaíso

\begin{tabular}{ccccc}
\hline $\begin{array}{c}\text { Distancia desde el conjunto social } \\
\text { a la quebrada más cercana } \\
\text { [metros] }\end{array}$ & $\begin{array}{c}\text { Cantidad de conjuntos } \\
\text { sociales por intervalo }\end{array}$ & $\begin{array}{c}\text { Cantidad de departa- } \\
\text { mentos / viviendas por } \\
\text { intervalo }\end{array}$ & $\begin{array}{c}\text { Porcentaje de la cantidad } \\
\text { de viviendas en el total } \\
\text { de viviendas }(\%)\end{array}$ & $\begin{array}{c}\text { Porcentaje } \\
\text { acumulado }\end{array}$ \\
\hline $0-50$ & 9 & 1734 & 16,58 & 16,58 \\
$50-100$ & 21 & 3797 & 36,30 & 52,88 \\
$100-150$ & 6 & 2437 & 23,30 & 76,18 \\
$150-200$ & 6 & 1355 & 12,95 & 89,13 \\
$200-250$ & 1 & 27 & 0,26 & 89,39 \\
$300-350$ & 2 & 89 & 0,85 & 90,24 \\
$400-450$ & 1 & 90 & 0,86 & 91,10 \\
$450-500$ & 1 & 46 & 0,44 & 91,54 \\
$550-600$ & 1 & 80 & 0,76 & 92,30 \\
$600-650$ & 4 & 390 & 3,73 & 96,03 \\
$700-750$ & 1 & 79 & 0,76 & 96,79 \\
$800-850$ & 1 & 64 & 0,61 & 97,40 \\
$850-900$ & 1 & 16 & 0,15 & 97,55 \\
$900-950$ & 1 & 66 & 0,63 & 98,18 \\
$1100-1150$ & 1 & 190 & 1,82 & 100,00 \\
Total general & 57 & 10460 & $100 \%$ & \\
\hline
\end{tabular}

Fuente: elaboración propia. 
e intervenidos constantemente en la búsqueda de posicionar el paradigma de la época que entre las décadas de 1920 a 1960 constituían suelos de baja calidad (Karzulovic, 1960); y, hacia fines del siglo XX se convirtieron en zonas protegidas por la vía normativa (Osorio et al. 2015).

No obstante, en virtud de las Leyes de Indias que normaron los emplazamientos urbanos coloniales, se contemplaban la construcción excluyentemente en terrenos secos, cercano a cursos de agua, mejorando el acceso al recurso, pero alejado de sistemas acuáticos considerados no higiénicos, como el caso de los humedales. Por lo mismo, la naturaleza o la perspectiva respecto de la naturaleza frente a ellos estuvo largo tiempo asociada a fuente de enfermedades. La cercanía con el sistema fluvial, si bien representaba la idea mayúscula de ciudad en progreso, sitios de emplazamiento industrial con viviendas asociadas a la matriz productiva -hoy consideradas patrimoniales- $\mathrm{y}$ el humedal interior próximo al centro de la ciudad, correspondían a una forma de paisaje abyecto, invisibilizado por los intereses productivos y abandonados por las políticas públicas (Fogle, 1962).

Con el terremoto de 1960, donde el terreno sufrió un basculamiento significativo, el humedal cobró más vigencia y protagonismo espacial, pasando a ser silenciado a costa de rellenos permanentes, los que permitieron la inclusión de numerosos conjuntos de vivienda popular. Esta situación telúrica que se reiteró con ocasión del terremoto de 2010 en la ciudad de Concepción y alrededores, donde particulares humedales desaparecieron por efecto del sismo (Quezada et al. 2012). Sin poder elegir el sitio más apto para construir, la vivienda social fue capturando estas intersticiales ventajas espaciales, que también terminarían por convertirse en un salvavidas de plomo para sus habitantes.

El desplazamiento de las ideas respecto de la naturaleza también ha permitido abrazar un proceso emancipador para las comunidades, y es así como lo comenta Skewes (et al. 2012) al abordar la lucha de un sector aledaño a un humedal por recuperarlo del proceso de degradación creciente al que estaba siendo sometido. De la misma forma, Rodríguez (2013) comenta el proceso intersubjetivo que se produce con los lugares donde la naturaleza dota de sentido a la ciudad de Valdivia, precisamente por su condición potencial de convertirse en espacio, no bien terminado en el paisaje ni en la imaginación, sino en trazos de construcción continua. Así como acontece con esta nueva construcción social que anticipa un uso inmobiliario funcional a las ventajas del río, hoy ocupado plenamente, se abre una brecha para el desarrollo inmobiliario dentro de los humedales.

\section{Encima de los humedales se reproduce el capital y la propiedad}

Para el caso de Valdivia, el índice global fue obtenido como la suma simple del índice de vivienda, educación y de elementos del hogar, para luego contrastarlo solo con el de vivienda y su proximidad con los humedales, como se expone en la siguiente figura.

La mayor concentración de valores altos del IDS está proyectado por la extensión de los bordes fluviales, particularmente en las zonas centrales de la ciudad. Esta forma no es esencialmente lejana, pues ya a principios del siglo XX estas tendencias eran evidentes y conformes al desarrollo manufacturero que Valdivia experimentó (Bernedo, 1999). De hecho, ha sido sobre estas bases que las posteriores urbanizaciones se han constituido en la zona, toda vez que ellas ocupan el espacio que las industrias despreciaron, ya sea por la finalización de su ciclo o por la fuga de los capitales hacia otras latitudes (Almonacid, 2000).

En este sentido, hacia el sur de la zona urbana valdiviana se encuentra una mayor dispersión del IDS vivienda, que expresa la posición mayoritaria de los conjuntos sociales hacia sectores distantes a los ríos que componen el sistema fluvial de Valdivia, como lo muestra la Figura 5.

A partir de la distancia euclidiana desde las manzanas al humedal más cercano, se obtiene una tendencia similar a la apreciaba en Valparaíso y sus quebradas, donde la mayor concentración para los guarismos menores en puntuación del IDS de vivienda se localizan más cerca de los humedales, como lo muestra la Tabla 4. Es importante reiterar que estos cuerpos de agua o zonas de inundación representaron, históricamente, los suelos de peor calidad en la ciudad. Esto ya lo indica Almonacid para los tiempos posteriores al incendio de 1909 donde las residencias de las clases populares, consumidas por el fuego, se trasladaron por iniciativa propia hacia los pantanos del sur de la ciudad (2000:84).

El número de conjuntos sociales alcanza las 48 unidades. El IDS global proyectado solo en los conjuntos sociales ilustra una propensión hacia una 


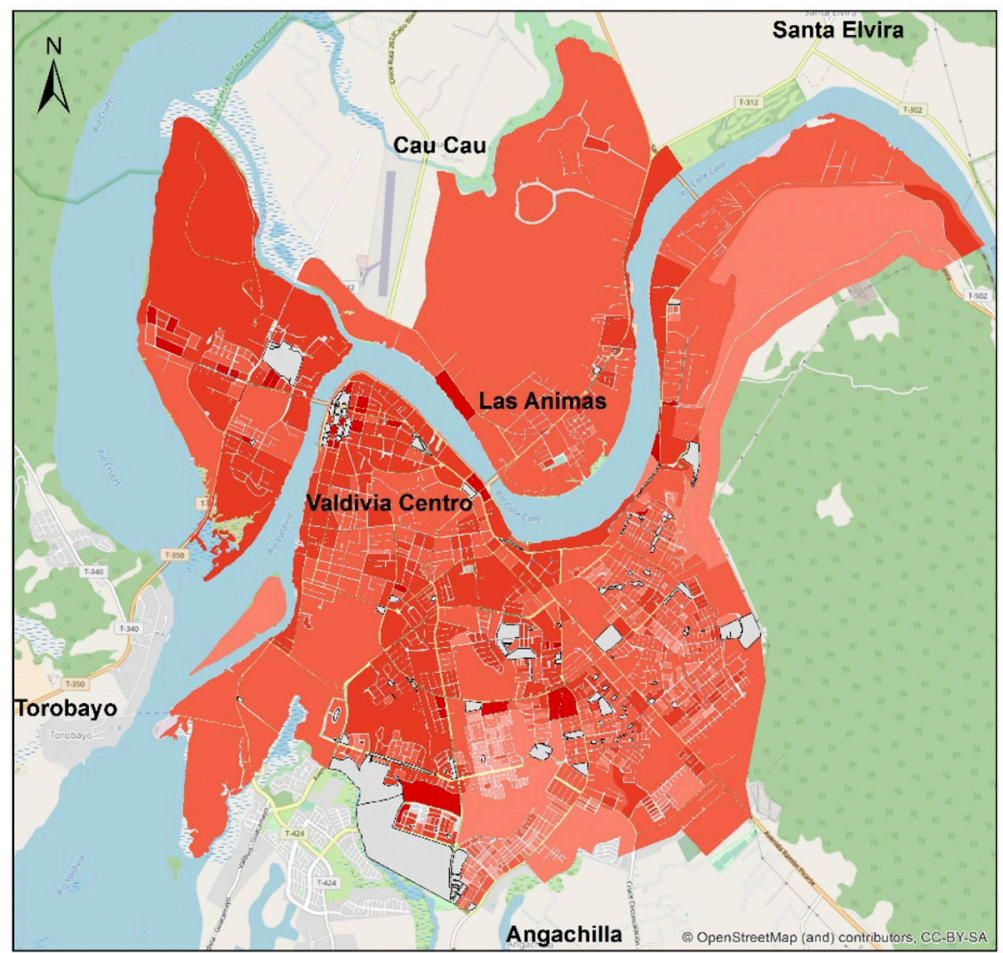

\begin{tabular}{|c|c|c|}
\hline \multicolumn{3}{|c|}{ Leyenda } \\
\hline \multicolumn{3}{|c|}{$\begin{array}{l}\text { Manzanas Valdivia } \\
\text { IDS_GLOBAL }\end{array}$} \\
\hline & 0,111111 & 00000 \\
\hline & 0,500001 & 000000 \\
\hline & 1,000001 & 500000 \\
\hline & 1,500001 & 000000 \\
\hline & 2,000001 & 500000 \\
\hline & 2,500001 & 000000 \\
\hline & Manzana & informacion \\
\hline $\begin{array}{l}\text { Datc } \\
\text { obte } \\
\text { Datc } \\
\text { Bas } \\
\text { Map }\end{array}$ & $\begin{array}{l}\text { Manzana } \\
\text { do con el so } \\
\text { Cartográfic } \\
\text { Cartográfic }\end{array}$ & $\begin{array}{l}\text { Censo } 2002 \\
\text { are Redatam } \\
\text { Eenso } 2002 \text {. } \\
\text { Open Street }\end{array}$ \\
\hline $\begin{array}{l}\text { Esc } \\
\text { Dati } \\
\text { Proy }\end{array}$ & $\begin{array}{l}1: 50.000 \\
\text { : WGS } 84 \\
\text { cción: UTM }\end{array}$ & \\
\hline 0 & 1.250 & $\begin{array}{l}2.500 \\
\text { Metros }\end{array}$ \\
\hline
\end{tabular}

Figura 4. Distribución del IDS Global en Valdivia.

Fuente: elaboración propia con base en datos IDE-Chile 2017.

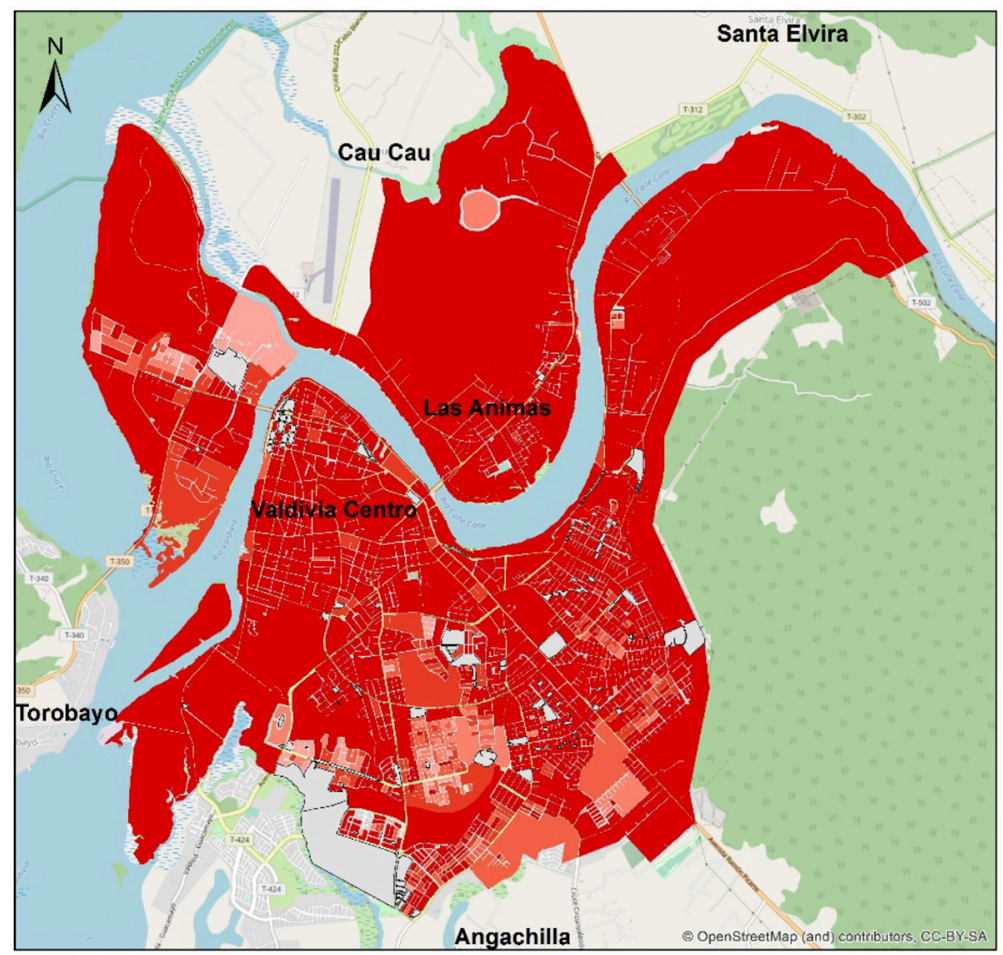

\section{Leyenda \\ Manzanas Valdivia IDS VIVIENDA}

\begin{tabular}{l}
0,000000 \\
$0,000001-0,200000$ \\
$0,200001-0,400000$ \\
$0,400001-0,600000$ \\
$0,600001-0,800000$ \\
$0,800001-1,000000$ \\
\hline$\quad$ Manzanas sin informacion
\end{tabular}

Datos Manzana: Censo 2002 obtenido con el software Redatam Datos Cartográficos: Censo 2002. Base Cartográfica: Open Street Map

Escala: 1: 50.000

Datum: WGS84

Proyección: UTM $18 S$

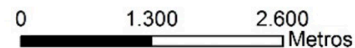

Figura 5. Distribución IDS de vivienda, Valdivia.

Fuente: elaboración propia con base en datos IDE-Chile 2017. 
Tabla 4. Promedio IDS Global por manzana censal e IDS por vivienda respecto de los humedales, Valdivia

\begin{tabular}{ccc}
\hline $\begin{array}{c}\text { Distancia desde las manzanas hacia } \\
\text { el humedal más cercano [m] }\end{array}$ & $\begin{array}{c}\text { Promedio } \\
\text { IDS Global }\end{array}$ & $\begin{array}{c}\text { Promedio } \\
\text { IDS VIVIENDA }\end{array}$ \\
\hline $0-100$ & 1,630 & 0,844 \\
$100-200$ & 1,663 & 0,815 \\
$200-300$ & 1,632 & 0,812 \\
$300-400$ & 1,772 & 0,826 \\
$400-500$ & 1,904 & 0,904 \\
$500-600$ & 1,979 & 0,876 \\
$600-700$ & 1,933 & 0,892 \\
$700-800$ & 1,814 & 0,808 \\
$800-900$ & 1,769 & 0,842 \\
$900-1000$ & 1,721 & 0,853 \\
$1000-1100$ & 1,757 & 0,828 \\
$1100-1200$ & 1,808 & 0,849 \\
$1200-1300$ & 1,737 & 0,797 \\
$1300-1400$ & 1,709 & 0,769 \\
$1400-1500$ & 1,829 & 0,830 \\
$1500-1600$ & 1,826 & 0,907 \\
$1600-1700$ & 1,784 & 0,882 \\
$1700-1800$ & 1,622 & 0,853 \\
$1800-1900$ & 1,638 & 0,908 \\
$1900-2000$ & 1,799 & 0,958 \\
$2000-2100$ & 1,635 & 0,982 \\
\hline
\end{tabular}

Fuente: elaboración propia con base en datos Censo 2002/bases censales 2016.

mayor concentración inmediata a los humedales, donde precisamente el IDS es menor al presentado en la figura anterior, esta vez respecto del sistema fluvial valdiviano. Sin embargo, la lógica de expansión sobre los humedales corresponde a una perspectiva a 2016, sin considerar aquellos sectores que fueron rellenados anteriormente en favor de la expansión habitacional, cuyo inventario no es conocido, salvo por ciertas fuentes que indican una masiva presencia de ellos en la ribera sur del río Valdivia, advirtiéndose su presencia con proyección hacia el centro de la ciudad (Fogle, 1962:17). Todos estos humedales fueron intervenidos con posterioridad al terremoto de 1960, generando un nicho de expansión habitacional sobre las zonas despreciadas, el que a la fecha parece como consolidado, como lo enseña la Figura 6.

Asemejándose al caso de Valparaíso, la densidad de conjuntos de habitaciones sociales en los primeros 500 metros lineales respecto de los humedales refleja un intenso proceso de producción de naturaleza en favor de la expansión urbana. Si bien la vocación histórica de rellenos en Valdivia opera constantemente sobre los humedales, la situación reafirma la ocupación de los sitios mayoritariamente por población de menores recursos. Sin embargo, y como se destaca en líneas anteriores, el valor paisajístico que posee para otros tipos de vivienda e ingresos, como lo reflejan los indicadores del IDS de vivienda, no significa una mayor integración espacial entre clases sociales. Es, simplemente, el valor de lo natural para algunos; y el cruel destino para otros. La tabla siguiente -número 5-caracteriza acusadamente este análisis.

Desde este cuadro se pueden apreciar tres sentencias claves para la revisión del caso valdiviano. La primera es que, sobre el humedal convertido en suelo urbanizable mediante el relleno, se presenta la vivienda social en considerable densidad. Segundo, al avanzar hacia el interior alejándose de los humedales, la tendencia apunta al aumento del valor por unidad residencial, hecho constatable en la Figura 6 y que grafica en la tabla que la amenidad forma parte de la renta extraída desde la producción de naturaleza. Finalmente, como tercer punto, la vivienda social de mayor antigüedad se localiza hacia el interior, pero que de todas formas se aproxima de forma desconcentrada y particular sobre otros humedales, ya totalmente intervenidos hacia el centro de Valdivia. 

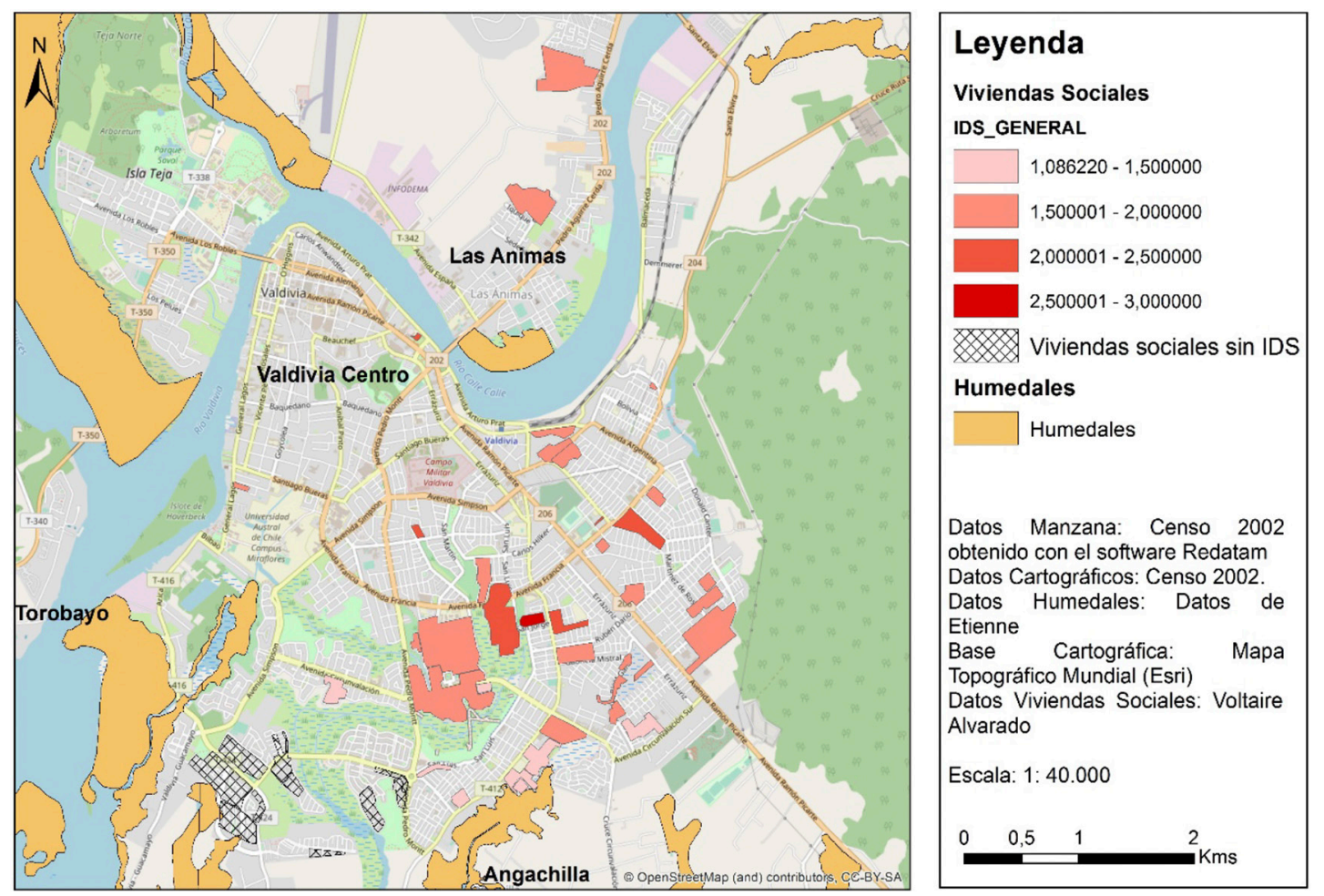

Figura 6. Distribución IDS, humedales y conjuntos de viviendas sociales, Valdivia.

Fuente: elaboración propia con base en datos IDE-Chile 2017.

Tabla 5. Relación de distancia y densidad de conjuntos habitacionales respecto de humedales, Valdivia.

\begin{tabular}{ccccc}
\hline $\begin{array}{c}\text { Distancia desde el conjunto } \\
\text { social a humedales más cercanos } \\
\text { [metros] }\end{array}$ & $\begin{array}{c}\text { Cantidad de conjuntos } \\
\text { sociales por intervalo }\end{array}$ & $\begin{array}{c}\text { Cantidad de departamentos } \\
\text { / viviendas por intervalo }\end{array}$ & $\begin{array}{c}\text { Porcentaje de la cantidad } \\
\text { de viviendas en el total } \\
\text { de viviendas }(\%)\end{array}$ & $\begin{array}{c}\text { Porcentaje } \\
\text { acumulado }\end{array}$ \\
\hline $0-50$ & 6 & 1426 & 13,71 & 13,71 \\
$50-100$ & 4 & 370 & 3,56 & 17,27 \\
$150-200$ & 2 & 259 & 2,49 & 19,76 \\
$250-300$ & 1 & 150 & 1,44 & 21,20 \\
$300-350$ & 1 & 56 & 0,54 & 21,74 \\
$350-400$ & 2 & 345 & 3,32 & 25,05 \\
$400-450$ & 1 & 150 & 1,44 & 26,49 \\
$450-500$ & 2 & 387 & 3,72 & 30,22 \\
$500-550$ & 4 & 594 & 5,71 & 35,93 \\
$550-600$ & 3 & 299 & 2,87 & 38,80 \\
$600-650$ & 3 & 969 & 9,32 & 48,12 \\
$650-700$ & 3 & 608 & 5,85 & 53,96 \\
$700-750$ & 4 & 437 & 4,20 & 58,16 \\
$800-850$ & 1 & 28 & 0,27 & 58,43 \\
$850-900$ & 4 & 452 & 4,35 & 62,78 \\
$950-1000$ & 1 & 150 & 1,44 & 64,22 \\
$1000-1050$ & 4 & 1390 & 13,36 & 77,58 \\
$1100-1150$ & 1 & 80 & 0,77 & 78,35 \\
$1150-1200$ & 2 & 255 & 2,45 & 80,80 \\
$1250-1300$ & 2 & 247 & 2,37 & 83,18 \\
$1300-1350$ & 2 & 368 & 2,15 & 85,33 \\
$1400-1450$ & 3 & 3,54 & 88,87 \\
\hline & & & & \\
\hline
\end{tabular}


Continuación Tabla 5.

\begin{tabular}{|c|c|c|c|c|}
\hline $\begin{array}{c}\text { Distancia desde el conjunto } \\
\text { social a humedales más cercanos } \\
\text { [metros }]\end{array}$ & $\begin{array}{l}\text { Cantidad de conjuntos } \\
\text { sociales por intervalo }\end{array}$ & $\begin{array}{l}\text { Cantidad de departamentos } \\
\text { / viviendas por intervalo }\end{array}$ & $\begin{array}{c}\text { Porcentaje de la cantidad } \\
\text { de viviendas en el total } \\
\text { de viviendas }(\%)\end{array}$ & $\begin{array}{l}\text { Porcentaje } \\
\text { acumulado }\end{array}$ \\
\hline $1450-1500$ & 1 & 76 & 0,73 & 89,60 \\
\hline $1500-1550$ & 3 & 528 & 5,08 & 94,67 \\
\hline $1550-1600$ & 1 & 50 & 0,48 & 95,15 \\
\hline $1750-1800$ & 1 & 60 & 0,58 & 95,73 \\
\hline $1900-1950$ & 1 & 444 & 4,27 & 100,00 \\
\hline Total general & 63 & 10402 & 100 & \\
\hline
\end{tabular}

Fuente: elaboración propia.

\section{Consideraciones finales}

La producción como proceso no es aséptico, de la misma forma que tampoco podría serlo la propiedad o los mecanismos de gestión para la vivienda social. Los conjuntos habitacionales localizados arriba de los cerros o sobre los humedales explican la bondad que el capital tiene para reproducirse más allá de los límites que el medio natural le impone. Aquellas zonas despreciadas como espacios proscritos para el desenvolvimiento del progreso son la matriz de crecimiento para ciudades en condiciones de sitio excepcionales, como es el caso de las descritas. Planteado de la forma en que lo considera Neil Smith, lo social es natural y lo natural es social, ecuación que es posible solo por la necesidad de construir espacio y propiedad, sin considerar la exposición y expoliación que estas dimensiones encierran.

Pero ¿cuál sería el acápite oculto en todo esto? A la vista de las evidencias, es la condición de sitio que aporta en la construcción social del valor en el espacio y que, por tanto, configura la relación entre renta y producción de naturaleza. Ningún Estado latinoamericano contemporáneo ha renunciado al fomento de la propiedad, y bajo este impulso es que se permite intervenir más allá de la capacidad misma de lo natural. Rellenar humedales como práctica de adaptación al medio, en un contexto de supervivencia en carencia de tecnologías, como los escenarios prehispánicos descritos por Adán (et al. 2017), pueden ser perfectamente replicados en la era del neoliberalismo de Estado que prima en la actualidad.

Las quebradas en Valparaíso presentan una ambivalencia en su valor de uso, algo que se reitera en Valdivia. Su valor como unidad de paisaje aporta a la construcción de diversas concepciones de valor, subjetivas y materiales, que justifican la necesidad ilimitada de ocupación espacial de zonas que, por complejas de convertir a los usos habitacionales, siempre están disponibles a la transformación en favor de la acumulación y la renta de los nichos grandes, erigidos por la necesidad del habitar.

\section{Referencias Citadas}

Adán Alfaro, L.; Urbina Araya, S. y Alvarado Pérez, M.

2017 "Asentamientos humanos en torno a los humedales de la ciudad de Valdivia en tiempos prehispánicos e históricos coloniales". Chungará, Revista de Antropología Chilena, 49(3): 1-19. DOI dx.doi.org/10.4067/S0717-73562017005000020. Aliste, E. y Musset, A.

2014 "Pensar los territorios del desarrollo: sustentabilidad y acción pública en nombre de una ciudad imaginaria. Concepción (Chile), 1950-2010". Revista EURE, 40(120):91-110.

Almonacid, F.

2000 "Ideas y proyectos en torno a la vivienda obrera en la ciudad de Valdivia 1900-1940". Revista de Ciencias Sociales, 4:81-114. Universidad Austral de Chile.
Bernedo, P.

1999 "Los industriales alemanes de Valdivia". Historia, 32:5.42. Instituto de Historia, Pontificia Universidad Católica de Chile.

Borsdorf, A. e Hidalgo, R.

2013 "Mountains and the sea: an analysis of the 2010 real estate and retail sectors in the Metropolitan Region of ValparaisoViña del Mar, Chile". Mountain Research and Development, 33(3):332-338. DOI 10.1659/MRD-JOURNAL-D-11-00080.1. CELADE/CEPAL.

2013 El uso y aplicaciones de Redatam+SP para la toma de decisiones en municipios y corporaciones municipales de desarrollo social, educación y salud. CELADE, Santiago de Chile. 
Engels, F.

2015 [1873] Sobre a questão da moradia. Boitempo Editorial, São Paulo.

Engels, F.

2017 [1971] Diálectica de la naturaleza. Akal, Madrid.

Fogle, D.

1962 City planning in Valdivia. The Valdivia City plan. Agency for International Development: Santiago de Chile.

Hidalgo, R.; Camus, P.; Alvarado, V.; Paulsen-Espinoza, A. y Olea, J.

2016 "Aguas de ficción a la carta: la producción de naturaleza como nicho de renta. Bienes comunes y espacio urbano exclusivo en torno a las crystal lagoons". En Expresión territorial de la fragmentación y segregación, coordinado por Concepción Alvarado, Ricardo Gómez y Rodrigo Hidalgo, pp. 13-30. Praxis Digital, Cuernavaca.

Hidalgo, R.; Alvarado, V. y Santana, D.

2017 "La espacialidad neoliberal de la producción de vivienda social en las áreas metropolitanas de Valparaíso y Santiago (1990-2014): ¿hacia la construcción ideológica de un rostro humano?". Cadernos Metrópole, 19(39):513-535. DOI dx.doi.org/10.1590/cm.v19i39.30179.

Gray de Cerdán, N.

1987 Territorio y Urbanismo. Bases de Geografía Prospectiva. CONICET, Mendoza.

Indiri, M. y Apablaza Minchel, S.

2010 "Protección del patrimonio en Valparaíso (Chile): proyecto 'MAR VASTO'”. Revista Ingeniería de Construcción, 25(1):21-62.

Jaramillo, S.

2001 "Acerca de la investigación en mercados de tierra urbana en América Latina". En Teorías sobre la ciudad en América Latina, compilado por Blanca Ramírez Velázquez y Emilio Pradilla Cobos, Vol. II, pp. 281-318. Universidad Autónoma Metropolitana, Iztapalapa, Ciudad de México.

Karzulovic, J.

1960 Informe sobre el agua subterránea en la ciudad de Valdivia. Instituto de Geología de la Universidad de Chile: Santiago de Chile.

Kropotkin, $\mathrm{P}$.

2016 La ciencia moderna y la anarquía. Editorial La Malatesta, Santiago de Chile.
Lefebvre, $\mathrm{H}$.

2014 El pensamiento marxista y la ciudad. Ediciones Coyoacán, Ciudad de México.

Lovera, A.

2013 Enfoques de investigación sobre el capital inmobiliario y constructor y la producción de la ciudad en América Latina (s/f). http://www.ungs.edu.ar/catedrasur/wp-content/ uploads/2012/11/8_LOVERA_VF.pdf (17 octubre de 2017).

Marín, A.; Link, F. y Valenzuela, F.

2017 "Arriendo en propiedad: arraigo y vulnerabilidad residencial en el barrio Puerto de Valparaíso". INVI, 32(90):127-157. DOI dx.doi.org/10.4067/S0718-83582017000200127.

Marx, K. y Engels, F.

1998 [1894] El Capital. Tomo III. El proceso global de producción capitalista. Siglo XXI Editores, Madrid, España.

Osorio, E.; Becker, A.; Sepúlveda, C. y Zuloaga, R.

2015 "Sustainable housing in Chilean Patagonia: from greenhouse to dewelling". European Journal of Sustainable Development, 4(2):9-18. DOI 10.14207/ejsd.2015.v4n2p9.

PNUD-MIDEPLAN

2000 Indicadores de Desarrollo Humano Comunal. PNUDMIDEPLAN, Santiago de Chile.

Quezada, J.; Jaque, E.; Fernández, A. y Vásquez, D.

2012 "Cambios en el relieve generados como consecuencia del terremoto $\mathrm{Mw}=8,8$ del 27 de febrero de 2010 en el centrosur de Chile". Revista de Geografia Norte Grande, 53:35-55.

Reclus, E.

1875 "La Tierra y los hombres". Revista Europea de Madrid, Año II, Tomo IV, 70: 663-664.

Rodríguez, L.

2013 "La ciudad como fuente icónica de sentido: aproximaciones desde la cultura geográfica en Valdivia". Revista AUS, 14:15-18. DOI 10.4206/aus.2013.n14-04.

Skewes, J.; Rehbein, R. y Mancilla, C.

2012 "Ciudadanía y sustentabilidad ambiental en la ciudad: la recuperación del humedal Angachilla y la organización local en la Villa Claro de Luna, Valdivia, Chile". Revista EURE, 38(113):127-145.

Zunino, H.M. e Hidalgo, R.

2009 "Spatial and socioeconomic effects of social housing policies implemented in neoliberal Chile: the case of Valparaíso". Urban Geography, 30(5):514-542. DOI 10.2747/0272-3638.30.5.514.

\section{Nota}

1 Una de las situaciones polémicas más intensas que se han vivido en Chile, a propósito de la disposición de los datos y la toma de decisiones en función de ellos, está en el Censo de Población del año 2012. Debido a los errores en la captura de datos, como el tiempo destinado para ello o la falta de capacitación de las personas que participaron del trabajo de campo, el Censo fue declarado inválido en el año 2015, luego de evacuados informes nacionales e internacionales que recomendaron gestionar en los próximos años, un Censo abreviado realizado en mayo de 2017. Para conocer el detalle de los informes, se recomienda revisar los documentos disponibles en www.ine.cl [18 octubre 2017]. 